\title{
Progress in liquid biopsy: A possible role of neutrophils'
}

\author{
Feixin Liang ${ }^{1 *}$ and Tao Yu ${ }^{2}$ \\ ${ }^{1}$ Department of Oral and Maxillofacial Surgery, Hospital of Stomatology, Guangxi Medical University, Nanning, Guangxi, China
}

${ }^{2}$ Graduate School of Guangxi Medical University, Nanning, Guangxi, China

\begin{abstract}
Recently, the non-invasive and diagnostic tumor liquid biopsy technique has received widespread attention from scientists. Current clinical research focuses mainly on the detection of circulating tumor cells, circulating tumor DNA, and related tumor biomarkers. However, the problem of low sensitivity in tumor diagnosis has become more prominent. In response to this limitation, some scholars have proposed to predict the occurrence and development of tumors by detecting changes in a large number of non-tumor cells that are in contact with the tumor microenvironment. Specifically, a large number of infiltrating neutrophils are common in tumor microenvironments. Studies have confirmed that the neutrophil-associated products are closely related to tumor occurrence and development and can predict tumor development. Therefore, this article will review the current tumor liquid biopsy limitations and explore the possible role of neutrophils in early tumor detection with liquid biopsy.
\end{abstract}

\section{Introduction}

The real-time detection of tumor cells and their cell products, such as tumor bioprotein markers, using liquid biopsy is predictive of tumor occurrence, development, and therapeutic effects [1]. Recently, liquid biopsy research has focused on circulating tumor DNA (ctDNA) and related tumor biomarkers in the peripheral blood.

\section{Progress and problems in liquid biopsy}

Liquid biopsies are non-invasiveness, specific, and provide realtime dynamic observation. However, the amount of early tumor cells and their associated tumor bioproteins that enter the peripheral blood is extremely small. For example, recent studies have shown that the number of oncogenic mutations per milliliter of serum is often less than one copy, which is below the lower limit of current routine biopsies [2,3]. Therefore, the sensitivity of liquid biopsies limits its clinical applications. Challenges remain in improving the sensitivity of conventional liquid biopsy for the diagnosis of tumors, especially with respect to early tumors.

Two research directions are currently addressing the low sensitivity of liquid biopsies.

1. Combined detection of ctDNA genes and tumor-associated biomarkers in the peripheral blood.

In early 2018, Science published the results of a breakthrough liquid biopsy method [4]. Eight common cancers, including lung and gastric cancer, can be diagnosed at one time by the combined detection of ctDNA gene mutations and eight common tumor-associated biomarkers in the peripheral blood. The median sensitivity for the eight cancers using this method reached $70 \%$. However, Jocelyn Kaiser's later Science publication [5], commented that the 1005 patients with cancer who participated in this study all had clinical symptoms, and of these patients, $45 \%$ had early stage cancers, which typically present no symptoms. Furthermore, the peripheral blood of such patients would have lower levels of tumor cells and tumor-associated biomarkers, and as such, the sensitivity of this method for patients with early-stage cancer patients needs to be further investigated across a large collection of clinical samples.

2. Detection of a large number of non-tumor cells in the peripheral blood.

This approach involves the detection of non-tumor cells that had been in contact with tumor cells and carry tumor information from the local tumor microenvironment to peripheral blood. Pantel et al. [6], found that in the tumor microenvironment, platelet RNA information changes after tumor contact, and thus conveys the presence of tumor biomarkers in the peripheral blood.

For example, in 2015, Best et al. [7], analyzed 283 clinical peripheral blood platelet samples and used platelet mRNA sequencing to diagnose tumors with $96 \%$ sensitivity. In this report, the amount of platelet mRNA that was required was only $100-500 \mathrm{pg}$. Although the mechanism of platelet-to-tumor changes in the peripheral blood after contact with tumor cells remains unclear, the study has the disadvantage of having a small sample size, and the presence of platelet mRNA is unstable over 48 hours [6], such studies have confirmed that normal cells in the peripheral blood that have been in contact with tumor cells in the tumor microenvironment facilitate early tumor diagnosis.

\section{The possible role of neutrophils in liquid biopsy}

In addition to platelets, a large number of infiltrating neutrophils are also present in local cancer tissues. This manifestation is common seen in tumor pathologies. Neutrophils account for 50\%-70\% of total leukocytes in the peripheral blood $\left(4.0-10.0 \times 10^{\wedge} 9 / \mathrm{L}\right)$. It is generally considered [7-9], that during the development of various cancers,

${ }^{*}$ Correspondence to: Feixing Liang, Department of Oral and Maxillofacial Surgery, Hospital of Stomatology, Guangxi, Medical University, Nanning, Guangxi, China, Tel: +86 771532 5160; E-mail: Liangfx@hotmail.com

Key words: tumor biomarkers, microenvironments, neutrophils, liquid biopsy

Received: May 04, 2018; Accepted: May 22, 2018; Published: May 28, 2018 
including oral cancer, inflammatory factors that are released by tumor cells, including reactive oxygen species and IL-8, may recruit peripheral blood neutrophils to local tumor microenvironments. This may play important roles in promoting tumor proliferation and metastasis $[10,11]$.

In reviewing current studies on the role of neutrophils in cancer growth and metastasis, the promise of neutrophil function as a biomarker has received special attention. Clinically, tumor neutrophil infiltration is associated with poor metastasis and prognosis [12], mainly in renal cancer, melanoma, liver cancer, colorectal cancer, lung cancer, and head and neck cancer [13]. Wang et al. [14], found that neutrophil infiltration in squamous cell carcinoma of the tongue is associated with lymph node metastasis, clinical stage, and increased tumor recurrence risk. However, the mechanism of neutrophil infiltration is not yet clear, and neutrophil counts within the tumor area can only be performed after surgery or tumor biopsy. Recently, preoperative and postoperative evaluations of the neutrophil to lymphocyte ratio in peripheral blood of patients with renal carcinoma and liver carcinoma have shown that the ratio of neutrophils to lymphocytes positively correlates with cancer recurrence [15-18]. However, the ratio of these two types of peripheral blood cells is often modified by virus infection and aplastic anemia. Whether the infiltrating neutrophils in tumor microenvironments can return to the peripheral blood and whether peripheral blood neutrophils are related to infiltrating neutrophils in the tumor microenvironment and tumor occurrence require further clarification.

The latest studies of neutrophils in inflammation show that neutrophils have the ability to accumulate from blood vessels to tissues and return from tissues to the peripheral blood [19-21]. Neutrophils have recently been shown to play important roles in tumor development in the tumor microenvironment, but the difference between neutrophils that return to peripheral blood after tumor microenvironment exposure and normal neutrophils remains unknown. Whether neutrophils can carry tumor information, migrate back into peripheral blood vessels, and then be tested through liquid biopsy also requires further clarification. A more in- depth and broader study of these issues will undoubtedly provide a new direction of tumor liquid biopsies.

\section{Summary}

- This study conducted the prevalence of GER and H. pylori infection in chronic pharyngolaryngitis patients.

- There was a significant higher association between GER and chronic pharyngolaryngitis.

- Sometimes patient is complaining of chronic cough or chocking for more than 3 monthes and he doesn't know that he has GER or $H$. pylori so ENT doctor has to investigate for them

- By good treatment for GER and H. pylori all the extra gastric symptoms will be improves.

\section{References}

1. Joosse SA, Pantel K (2015) Tumor-Educated Platelets as Liquid Biopsy in Cancer Patients. Cancer Cell 28: 552-554. [Crossref]
2. Bettegowda C, Sausen M, Leary RJ, Kinde I, Wang Y, et al. (2014) Detection of circulating tumor DNA in early-and late-stage human malignancies. Sci Transl Med 6: 224ra24-224ra24.

3. Cohen JD, Javed AA, Thoburn C, Wong F, Tie J, et al. (2017) Combined circulating tumor DNA and protein biomarker-based liquid biopsy for the earlier detection of pancreatic cancers. Proc Natl Acad Sci U S A 114: 10202-10207.

4. Cohen JD, Li L, Wang Y, Thoburn C, Afsari B, et al. et al. (2018) Detection and localization of surgically resectable cancers with a multi-analyte blood test. Science 359: 926-930. [Crossref]

5. Jocelyn K (2018) Liquid biopsy for cancer promises early detection. Science 359: 259 [Crossref]

6. Joosse SA, Pantel K (2015) Tumor-Educated Platelets as Liquid Biopsy in Cancer Patients. Cancer Cell 28: 552-554. [Crossref]

7. Kim J, Bae JS (2016) Tumor-associated macrophages and neutrophils in tumor microenvironment. Mediators Inflamm: 6058147. [Crossref]

8. Mishalian I, Bayuh R, Levy L, Zolotarov L, Michaeli J, et al. (2013) Tumor-associated neutrophils (TAN) develop pro-tumorigenic properties during tumor progression. Cancer Immunol Immunother 62: 1745-1756.

9. Jablonska J, Leschner S, Westphal K, Lienenklaus S, Weiss S (2010) Neutrophils responsive to endogenous IFN-beta regulate tumor angiogenesis and growth in a mouse tumor model. J Clin Invest 120: 1151-1164. [Crossref]

10. Galdiero MR, Varricchi G, Loffredo S, Mantovani A, Marone G (2018) Roles of neutrophils in cancer growth and progression. J Leukoc Biol 103: 457-464. [Crossref]

11. Felix K, Gaida MM (2016) Neutrophil-derived proteases in the microenvironment of pancreatic cancer-active players in tumor progression Int J Biol Sci 12: 302. [Crossref]

12. Donskov F (2013) Immunomonitoring and prognostic relevance of neutrophils in clinical trials. Semin Cancer Biol 23: 200-207. [Crossref]

13. Paneesha S, McManus A, Arya R, Scriven N, Farren T, et al. (2010) Frequency, demographics and risk (according to tumour type or site) of cancer-associated thrombosis among patients seen at outpatient DVT clinics. Thromb Haemost 103: 338343. [Crossref]

14. Wang N, Feng Y, Wang Q, Liu S, Xiang L5 et al. (2014) Neutrophils infiltration in the tongue squamous cell carcinoma and its correlation with CEACAM1 expression on tumor cells. PLoS One 9: e89991. [Crossref]

15. Ohno Y, Nakashima J, Ohori M, Hatano T, Tachibana M (2010) Pretreatment neutrophil-to-lymphocyte ratio as an independent predictor of recurrence in patients with nonmetastatic renal cell carcinoma. J Urol 184: 873-878. [Crossref]

16. Motomura T, Shirabe K, Mano Y, Muto J, Toshima T, et al. (2013) Neutrophillymphocyte ratio reflects hepatocellular carcinoma recurrence after liver transplantation via inflammatory microenvironment. J Hepatol 58: 58-64. [Crossref]

17. Ohno Y, Nakashima J, Ohori M, Gondo T, Hatano T, et al. (2012) Followup of neutrophil-to-lymphocyte ratio and recurrence of clear cell renal cell carcinoma. J Urol 187: 411-417. [Crossref]

18. Lai Q, Castro Santa E, Rico Juri JM, Pinheiro RS, Lerut J (2014) Neutrophil and platelet to-lymphocyte ratio as new predictors of dropout and recurrence after liver transplantation for hepatocellular cancer. Transpl Int 27: 32-41. [Crossref]

19. Wang J, Hossain M, Thanabalasuriar A, Gunzer M, Meininger C, et al. (2017) Visualizing the function and fate of neutrophils in sterile injury and repair. Science 358: 111-116. [Crossref]

20. Colom B, Bodkin JV, Beyrau M, Woodfin A, Ody C, et al. (2015) Leukotriene B4Neutrophil Elastase Axis Drives Neutrophil Reverse Transendothelial Cell Migration In Vivo. Immunity 42: 1075-1086. [Crossref]

21. Hamza B, Irimia D (2015) Whole blood human neutrophil trafficking in a microfluidic model of infection and inflammation. Lab Chip 15: 2625-2633. [Crossref]

Copyright: (C2018 Liang F. This is an open-access article distributed under the terms of the Creative Commons Attribution License, which permits unrestricted use, distribution, and reproduction in any medium, provided the original author and source are credited. 